\title{
A case of emphysematous gastritis
}

A 71-year-old man was referred with food intolerance leading to a weight loss of $12 \mathrm{~kg}$. Three weeks earlier, he had presented at another hospital with severe upper abdominal pain and fever. He was treated with intravenous fluids and ciprofloxacin and recovered rapidly. CT imaging 3 weeks later showed mucosal edema of the stomach with air and fluid collections within the gastric wall ( Fig. 1). Esophagogastroduodenoscopy (Olympus GIF H180) showed significant edema of the gastric mucosa, creating a tunnel view leading to the pylorus. In the antrum, two ulcer-like lesions covered by pus were observed along the greater curvature. Intubation of these lesions with a small-caliber scope (Olympus GIFXP180N) showed loosening of the gastric mucosa, which had a puff-pastry appearance ( Video 1 ). This led us to a diagnosis of emphysematous gastritis. Gram staining of the aspirated pus showed the presence of gram-positive cocci and bacilli. A culture grew Candida albicans. Treatment with piperacillin-tazobactam, metronidazole, and fluconazole was started and the patient was fed by nasojejunal tube. He ultimately had a full recovery, gaining $20 \mathrm{~kg}$ in weight over the ensuing year.

\section{Video 1}

Esophagogastroduodenoscopy (Olympus GIF H180) showed significant edema of the gastric wall and the presence of ulcer-like lesions in the prepyloric region, covered by pus, and multiple small mucosal defects along the greater curvature. Endoscopy of the submucosal layers was subsequently performed through the largest of the prepyloric mucosal defects using a small-caliber scope (Olympus GIF XP180N), unrevealing a loosening of the gastric mucosa which had a puff-pastry appearance.

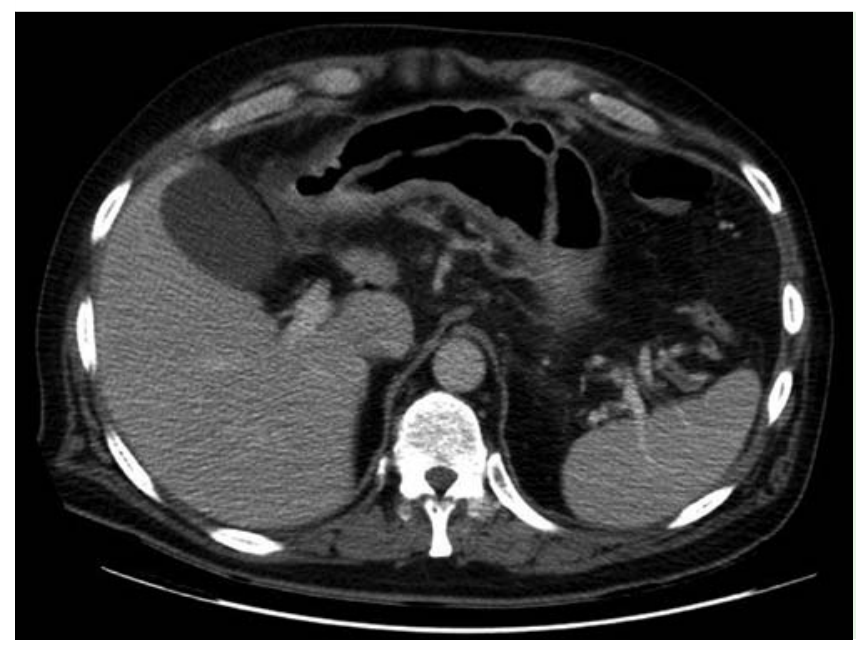

Fig. 1 CT scan shows a thickening of the gastric wall with large air inclusions.

Eighteen months later the patient presented again with postprandial vomiting and acid regurgitations accompanied by a rapid weight loss of $10 \mathrm{~kg}$. His C-reactive protein concentration was elevated. CT scan showed a thickening of the antral gastric wall with reappearance of air inclusions. A subtotal gastrectomy was performed. An impacted food granuloma was observed in the submucosal layers of the antrum, with surrounding inflammation and fibrosis.

Emphysematous gastritis is a rare disease with a poor overall prognosis; it is caused by invasion of the gastric wall by gasforming micro-organisms [1,2]. Patients who do not respond to conservative treatment or have severe necrosis should be considered for surgery. The follow-up of our case shows that ultimately a subtotal gastrectomy may be indicated despite an initial complete recovery with the conservative approach.

\section{Endoscopy_UCTN_Code_CCL_1AB_2AD_3AF}

Competing interests: None

\section{Verhelst, M. Cabooter, V. de Wilde, P. Laukens, H. Orlent}

Department of Gastroenterology and Hepatology, AZ St Jan AV Brugge, Belgium

\section{References}

1 Huang CT, Liao WY. Emphysematous gastritis: a deadly infectious disease. Scand J Infect Dis 2009; 41: 317-319

2 Moosvi AR, Saravolatz LD, Wong $D H$ et al Emphysematous gastritis: case report and review. Rev Infect Dis 1990; 12: 848-855

Bibliography

Dol http://dx.doi.org/

10.1055/s-0031-1291746

Endoscopy 2012; 44: E159

(c) Georg Thieme Verlag KG

Stuttgart · New York

ISSN 0013-726X

\section{Corresponding author}

\section{H. Orlent, MD}

Head of Department of Gastroenterology and Hepatology

AZ Sint Jan AV

Ruddershove 10

8000 Brugge

Belgium

Fax: +32-50-452179

Hans.Orlent@azsintjan.be 